\title{
Introduction of an ex-vivo pig model for teaching percutaneous nephrolithotomy access techniques
}

Connor M. Forbes ${ }^{1}$; Jonathan Limㄹ ${ }^{1}$ Justin Chan ${ }^{1}$; Ryan F. Paterson ${ }^{1}$; Mantu Gupta ${ }^{2}$; Ben H. Chew ${ }^{1}$; Kymora Scotland ${ }^{1}$

${ }^{1}$ Department of Urologic Sciences, University of British Columbia, Vancouver, BC, Canada;

${ }^{2}$ Mount Sinai West and St. Luke's Hospitals \& department of Urology, Icahn School of Medicine, Mount Sinai, New York, NY, United States

Acknowledgements: The ex-vivo partial pig organ training model was initially developed by the Cook Medical company as part of their hands-on training curriculum. The silicone training model was used during the training sessions also was borrowed from Cook through the efforts of their representatives. The authors thank Messrs. Charles Wiggins, Alex Szabo, and Brad Wittal of Cook Medical for their assistance with this project.

Cite as: Can Urol Assoc J 2019 February 7; Epub ahead of print. http://dx.doi.org/10.5489/cuaj.5717

Published online February 7, 2019

$* * *$

\section{Abstract}

Introduction: In North America, obtaining access for percutaneous nephrolithotomy (PCNL) is often not performed by urologists. Hands-on training sessions help to ensure this skill continues within the urologic community. An ex vivo pig kidney model was developed for simulation. This model uses porcine tissues with a fluoroscopic C-arm and standard PCNL equipment. The bullseye or triangulation techniques are both possible. We propose this as a high-fidelity tool for teaching PCNL access.

Methods: The pig kidney, fat, ribs, flank, and skin were arranged anatomically on a table with fluoroscopy. Hands-on training was provided to residents and urologists using the ex-vivo pig model and a silicone-based percutaneous access model. Questionnaires were given at the end of the session.

Results: There were 14 responders total for each model, with incomplete responses on two surveys. A total of $15 \%$ of responders for the pig model and $7 \%$ of responders for the silicone model had previous percutaneous access experience. For the pig model, 93\% of trainees agreed or strongly agreed that the model was easy to use, and $79 \%$ of the silicone model trainees felt the same. After the session, $50 \%$ of silicone model trainees and $86 \%$ of pig model trainees reported 
increased confidence in their ability to obtain PCNL access. All the pig model trainees and $71 \%$ of the silicone model trainees felt that the simulation activity was worthwhile.

Conclusions: The inexpensive but anatomically realistic ex-vivo pig model using real world equipment provides trainees with an excellent tool to learn PCNL access.

\section{Introduction}

Achieving appropriate percutaneous access is essential for efficient and successful percutaneous nephrolithotomy (PCNL). In North America, radiologists often obtain access as the first part of a two-stage procedure as opposed to urologists obtaining their own access. However, urologistobtained access can save time by transforming the operation into a single-stage procedure in the operating room. A previous study comparing urologist and radiologist obtained access found greater stone free rates when access was achieved by urologists ( $86 \%$ vs $61 \%) .{ }^{1}$ There is a steep learning curve in obtaining percutaneous access, which is an obstacle in urological training. Studies suggest that 60 PCNL procedures are required for residents to gain surgical competence. ${ }^{2}$ Meanwhile, residents are frequently graduating without competency in percutaneous access, with only $37.5 \%$ of Canadian residents reporting training in the procedure. ${ }^{3}$ Simulation models have been developed as training adjuncts. These include silicone models, live pig procedures, and virtual reality simulators.

These models can be used in low risk environments. They can function as adjuncts to surgical training in order to increase trainee exposure. Affordable silicone models exist, but are considered low-fidelity simulators. Sophisticated silicone models can simulate the procedure with greater fidelity but often require expensive setups. Virtual reality simulators have some benefit, but lack aspects of the percutaneous access procedure that hands-on training captures more accurately. Obtaining access on live pigs is costly and cumbersome, although it incorporates realistic motions that interfere with access such as respiration. At this time, the high-fidelity simulators for gaining PCNL access are associated with high costs of operation. To increase fidelity at a low price-point, a new model has been introduced that uses ex-vivo porcine organs. This arrangement shares some of the principles underlying the silicone model but increases fidelity by using real tissue. Furthermore, instead of embedding real organs in a silicone model, as performed by Strohmaier and Giese, ${ }^{4}$ this ex-vivo pig organ model includes tissue layers in the flank, abdomen and subcutaneous fat of the pig as well as the renal system. The set-up can be free standing on an operating table without any supporting equipment. The advantage of this arrangement is that trainers have more freedom in the set-up of the model. For example, the $11^{\text {th }}$ and $12^{\text {th }}$ ribs can be included if desired to increase the difficulty for trainees and to add to the realism of the model. Real-time fluoroscopy with a C-arm that is used clinically can be implemented in this training style. The bullseye and triangulation techniques to gain access in the prone position can both be performed. With its low cost and similarity to actual 
clinical experience, the $e x$-vivo pig model may be an accessible tool to teach and learn techniques for gaining PCNL access. The present study aims to compare the ex-vivo pig organ training model for prone percutaneous access to the more traditional silicone model through questionnaires administered to trainees exposed to both models.

\section{Methods}

A simulation model for learning and teaching percutaneous access was developed and evaluated. The model used for this study was previously developed by Dr. Gupta in collaboration with Cook Medical (Bloomingdale, IN) during their training courses. This model uses pig tissue and common PCNL equipment including a C-arm, contrast, access needles, catheters and guidewires. The tissue required is a whole pig kidney including attached ureter, pig flank including ribs, flank muscle, and pig skin with subcutaneous fat. A C-arm or comparable fluoroscopic imager with rotational capacity is also required, as is lead and personal protective equipment for the participants. All usual PCNL access equipment is needed, including needles for obtaining access, guidewires of preference, contrast dye, and syringes.

The model setup is illustrated in Figure 1. The setup is outlined as follows. The pig kidney is placed inside a slab of foam cut to accommodate the kidney and ureter inside a plastic tray, with the ureter and pelvis displayed in an orthotopic fashion. A 5F ureteric catheter (Pollock, Cook Medical, Bloomingdale IN) is placed in the pig ureter and secured with a suture, then attached to a syringe with diluted contrast for injecting into the collecting system. Overlying this, the flank steak is placed with ribs covering the upper pole of the kidney to simulate the position of ribs in vivo. Finally, a sheet of pig skin with subcutaneous tissue is laid overtop, anchored by its own weight. Any gaps between the ribs and flank are filled with rectangular foam blocks $2.5 \mathrm{~cm}$ thick. The $\mathrm{C}$-arm is then brought into position. From here, the usual bullseye or triangulation access techniques can be performed using the simulation model. Figure 2 shows percutaneous access being achieved on the model, while Figure 3 shows fluoroscopic images obtained during the process.

The ex-vivo pig organ model was used as part of a course for participants with little experience obtaining percutaneous access or being exposed to simulations. Trainees were taught percutaneous access techniques on a silicone PCNL model. The silicone model used was a proprietary model developed by Dr. Gupta with Cook Medical (Bloomingdale, IN). It is composed of a silicone block with a simulated collecting system within. The model is backlit to approximate obtaining fluoroscopic access (Fig 4). The pig-tissue simulation model was taught separately. Trainees reused a single pig model kidney for multiple punctures. There was no standardized order between the two models, with some trainees using one first and some using the other. A single trainee did not have exposure to both models. This was all due to time and logistical constraints during the sessions. The authors were not participants in the trials. An 8item questionnaire was distributed to the participants after using each simulation model to 
evaluate the model as a learning tool. These questions were scored on a 5-point Likert scale (1=strongly disagree, $5=$ strongly agree). Results were tabulated.

The sample size needed to detect a statistically significant difference between the models was calculated. Assuming normally distributed populations, a level of significance of 0.05 and $80 \%$ power, the minimum sample size that would have been needed to detect a $25 \%$ difference in the models is 250 participants. The Mann Whitney $\mathrm{U}$ test was used to compare outcomes between models.

\section{Results}

Responses to the questionnaires were obtained from 14 participants for the ex-vivo pig model and 14 participants for the silicone model. Participants included residents and trained urologists. One of the questionnaires for each model was incomplete with missing information on one question. Two of 13 responders (15\%, one non-responder) for the pig model and 1 of 14 responders for the silicone model (7\%) had previous experience obtaining percutaneous access. The model was felt to be easy to use by $93 \%$ of trainees in the pig model and $79 \%$ of the trainees in the silicone model.

All of the pig model trainees and $57 \%$ of the silicone model trainees reported that the model increased their understanding of the anatomy. However, only $23 \%$ of the trainees felt that the silicone model simulated what they would expect in a human patient, whereas $100 \%$ of the pig model trainees felt that the pig model replicated clinical experience. This was based on participant impression of fidelity, as many participants did not have previous human PCNL access experience. Both of these findings represented a statistically significant difference favouring the ex vivo pig model (Tables 1,2).

In terms of confidence with the procedure, $65 \%$ of the silicone model trainees felt that they had a better understanding of the steps of the procedure after the exercise. This was the case for all (100\%) of the pig model trainees. A total of $50 \%$ of silicone model trainees and $86 \%$ of pig model trainees felt more confident in their ability to obtain PCNL access. All the pig model trainees and $71 \%$ of the silicone model trainees felt that the simulation activity was worthwhile. Once again, responses to all three of these questions yielded a statistically significant difference favouring the ex vivo pig model. Results are summarized in Tables 1 and 2.

The cost of the ex vivo pig model was relatively low. The total cost was approximately \$216 (CDN) for one model, with individual component costs summarized in Table 3. It should be noted that this is the approximate cost for one participant. Additional participants cost an additional \$20. This is the price for an additional pig kidney, with the rest of the model reusable on the same day. For example, the total cost for 2 participants would be $\$ 236$, and the total cost for 10 participants would be $\$ 396$, allowing for approximately 5 punctures per participant. 


\section{Discussion}

Gaining PCNL access remains an important skill for urologists. Competency in this procedure requires an extensive learning curve. An increasing number of urologists are not being trained in this procedure and instead rely on their interventional radiology colleagues to gain access. However, maintaining this skill set within the field of urology is beneficial to both patients and surgeons: it saves time in the operating room and may contribute to higher stone free rates. ${ }^{1}$ Particularly in light of the increasing demands for productivity in the operating room and costefficient patient care, the ability to independently obtain percutaneous entry allows the operation to become a faster single-stage procedure. In addition, radiologists are not available to perform this procedure at all institutions, which may lead to suboptimal or ultimately more expensive care for the patient and health system. Simulation models are useful for trainees, especially if clinical volume of urologist-obtained access at a given center is limited. Previously developed models include silicone, virtual reality, and living porcine models. Each of these has its benefits and drawbacks.

silicone models used for practicing PCNL access are physical representations of renal and perirenal anatomy. These vary in complexity. Some models are simple boxes with a visible collecting system on which trainees practice punctures. Recent models replicate human anatomy by using 3-D printing and the computed results of a computed tomography (CT) urogram. Fluoroscopy can be used on silicone models, some of which can endure up to 20 punctures without leaking contrast. ${ }^{5}$ Other models avoid the use of fluoroscopy. For example, the C-arm Trainer (CAT) processes the images from a camera to simulate fluoroscopy, ${ }^{6}$ which trainees can use to practice gaining access. While silicone models can be a cost-effective training model, they are not equivalent to an actual PCNL procedure. The synthetic material cannot give trainees the same tactile experience as gaining access through real tissue.

A more accurate representation of the human body is that of the living porcine model. In this model, trainees have the opportunity to practice by carrying out the procedure on a live pig under general anesthetic. The pig is positioned on the operating table with equipment and machinery comparable to those used in the OR, including guide wires, access needles, and the Carm. Fluoroscopy can be utilized, and access is gained through real tissue of a living organism. With the real-time pressure of anaesthesia, movement of the kidney, tactile feedback, as well as aspiration to confirm a successful puncture, ${ }^{7}$ this set-up is the most realistic option for trainees without actually operating on a human. Most notably, the use of real C-arm fluoroscopy, needles, and guidewires makes this a high-fidelity model. However, this immersive experience comes at a high financial cost because of the supporting equipment and personnel required. In addition to the significant cost of pig procurement, specialized veterinary staff are required to anaesthetize the pig and must be present throughout the procedure. ${ }^{7}$ Therefore, while this method of training closely simulates the actual procedure, it is difficult for trainees to access and use this model on a frequent basis. 
Virtual reality (VR) is a novel training model available in endourology. VR models create a virtual environment for trainees that mimic the real clinical scenario. The virtual experience encompasses the entire process of obtaining access including puncturing the pelvicalyceal system, aspiration from the puncture needle, and injecting contrast through the catheter. ${ }^{8}$ The training is also supplemented with a foot pedal to control a biplanar VR C-arm that can simulate fluoroscopy in a radiation-free environment. ${ }^{8}$ In addition to a realistic depiction of the actual surgery, the VR simulator has the added benefit of scoring the trainee on their performance. ${ }^{8}$ This allows trainees to identify exactly where errors occur. However, this training method is still lacking tactile feedback from real tissue and is also expensive, making it one of the less common training methods.

This study compares trainees' assessment of two low-cost models for percutaneous access, a silicone model and an ex-vivo pig organ model. The ex vivo pig organ model is attractive because its use of tissue increases fidelity. The texture of the tissue, the presence of ribs, and the ability to add contrast to the system is similar to obtaining access in humans. Other advantages of this system are the ease with which components can be procured inexpensively and the straightforward set-up. Our cost estimate for one participant on one model was CDN \$216. In addition, there are cost savings for a larger group as many components of the model are reusable. This is far less than the estimated cost of $\$ 1950$ for one live pig model at our institution, which includes anesthetic and veterinary technician time. The model can be arranged independently which makes it amenable for use in residency or community urologist training programs as part of a simulation training session. Multiple punctures are possible, with our experience showing unacceptable contrast extravasation after approximately 5 punctures. While both the ex-vivo pig model and the silicone model were found to be beneficial, $100 \%$ of participants found the ex-vivo model to be worthwhile versus $71 \%$ for the silicone model. Moreover, all participants agreed that the porcine model simulated human anatomy well, and $86 \%$ concluded that they had gained a better understanding of the steps for percutaneous access. These differences were statistically significant. Ultimately, the questionnaire results suggest the ex vivo pig model is a valuable addition to academic activities for urology residents as well as established urologists who are learning to obtain access. The sample size in this study fell beneath the number needed to detect a $25 \%$ statistically significant difference between populations. This indicates that non-statistically significant values may not have detected a difference of that size between the study populations.

Similar studies corroborate our findings. Häcker et al inserted a porcine kidney with attached ureter into a chicken carcass ${ }^{9}$ that was used in the same fashion as our partial pig model. Their model supported ultrasound and fluoroscopic guidance. It was found to be effective for teaching and skill acquisition for a variety of endourologic procedures. A major disadvantage of the chicken carcass was the visible skeleton under $\mathrm{x}$-ray ${ }^{9}$, obstructing the view of the collecting system in a manner not usually observed in human patients. Zhang et $\mathrm{al}^{10}$ and Strohmaier and 
Giese $^{11}$ also tested ex-vivo porcine models with positive results. Zhang et al wrapped a porcine kidney in a flap of full-thickness porcine skin with subcutaneous fascia and muscle and secured the model on a wooden board with two steel nails. ${ }^{10}$ Meanwhile, Strohmaier and Giese improved upon their porcine-silicone hybrid model ${ }^{4}$ to create a fully biologic model consisting of a porcine kidney embedded in a porcine thoracic/abdominal wall. ${ }^{11}$ Both studies reported effective training with their models.

While this ex-vivo partial pig model conveys substantial training benefits, it is not without its limitations. These include the need for a C-arm for fluoroscopy, which necessitates the use of a fluoroscopy-compatible table for the training session. Furthermore, the natural movement of the kidney caused by breathing is not present. ${ }^{9}$ Bleeding is also not present in the model. The model cannot be used for ultrasound guided access due to the air between the tissue layers. This is also a model for prone percutaneous access and is not currently suited for access with the patient in the supine position. Lastly, most participants in this study had minimal prior exposure to human PCNL access, making them less qualified to comment on the authenticity of the model as compared to obtaining access in humans. The ex vivo pig model was tested in a limited population, and evaluation in further populations and with alternative scoring instruments will be necessary to assess for general validity. ${ }^{12}$

\section{Conclusions}

Participant questionnaire results comparing a silicone model to an ex-vivo pig organ found a positive training experience with both models. Moreover, the ex-vivo partial pig model has a straightforward setup, inexpensive components and more faithfully replicates the surgical percutaneous nephrolithotomy procedure. Thus, training and practice for PCNL access can be meaningfully executed with this model.

Conflicts of interest: Ben Chew is a consultant for ADVA-TEC Inc, AURIS Surgical, Boston Scientific Corporation, Olympus and Cook Medical; and a lecturer for Bard Medical. All other authors have no conflicts of interest to disclose. 


\section{References}

1. Watterson JD, Soon S, Jana K. Access related complications during percutaneous nephrolithotomy: urology versus radiology at a single academic institution. The Journal of urology. 2006;176(1):142-145.

2. Tanriverdi O, Boylu U, Kendirci M, Kadihasanoglu M, Horasanli K, Miroglu C. The learning curve in the training of percutaneous nephrolithotomy. European urology. 2007;52(1):206-211.

3. Preston MA, Blew BD, Breau RH, Beiko D, Oake SJ, Watterson JD. Survey of senior resident training in urologic laparoscopy, robotics and endourology surgery in Canada. Canadian Urological Association journal = Journal de l'Association des urologues du Canada. 2010;4(1):42-46.

4. Strohmaier WL, Giese A. Ex vivo training model for percutaneous renal surgery. Urological research. 2005;33(3):191-193.

5. Turney BW. A new model with an anatomically accurate human renal collecting system for training in fluoroscopy-guided percutaneous nephrolithotomy access. Journal of endourology. 2014;28(3):360-363.

6. Noureldin YA, Hoenig DM, Zhao P, et al. Incorporation of the fluoroless C-Arm Trainer at the American Urological Association hands on training percutaneous renal access. World journal of urology. 2018;36(7):1149-1155.

7. Mishra S, Kurien A, Ganpule A, Muthu V, Sabnis R, Desai M. Percutaneous renal access training: content validation comparison between a live porcine and a virtual reality (VR) simulation model. BJU international. 2010;106(11):1753-1756.

8. Noureldin YA, Andonian S. Simulation for Percutaneous Renal Access: Where Are We? Journal of endourology. 2017;31(S1):S10-s19.

9. Hacker A, Wendt-Nordahl G, Honeck P, Michel MS, Alken P, Knoll T. A biological model to teach percutaneous nephrolithotomy technique with ultrasound- and fluoroscopy-guided access. Journal of endourology. 2007;21(5):545-550.

10. Zhang Y, Ou TW, Jia JG, et al. Novel biologic model for percutaneous renal surgery learning and training in the laboratory. Urology. 2008;72(3):513-516.

11. Strohmaier WL, Giese A. Improved ex vivo training model for percutaneous renal surgery. Urological research. 2009;37(2):107-110.

12. Noureldin YA, Lee JY, McDougall EM, Sweet RM. Competency-Based Training and Simulation: Making a "Valid" Argument. Journal of endourology. 2018;32(2):84-93. 


\section{Figures and Tables}

Fig. 1. Ex-vivo partial pig model setup. (a) Foam container with cut-out kidney impression. (b) Pig kidney with ureter placed on absorbent pad on foam. (c) Pig flank placed overtop of kidney. (d) Further pig flank slabs, including ribs placed overtop to simulate human anatomy. (e) Complete setup of partial pig model: final layer consists of skin and subcutaneous tissue. (f) $5 \mathrm{Fr}$ open-ended ureteric catheter is placed up ureter and tied in place, contrast syringe is attached to external end of catheter.
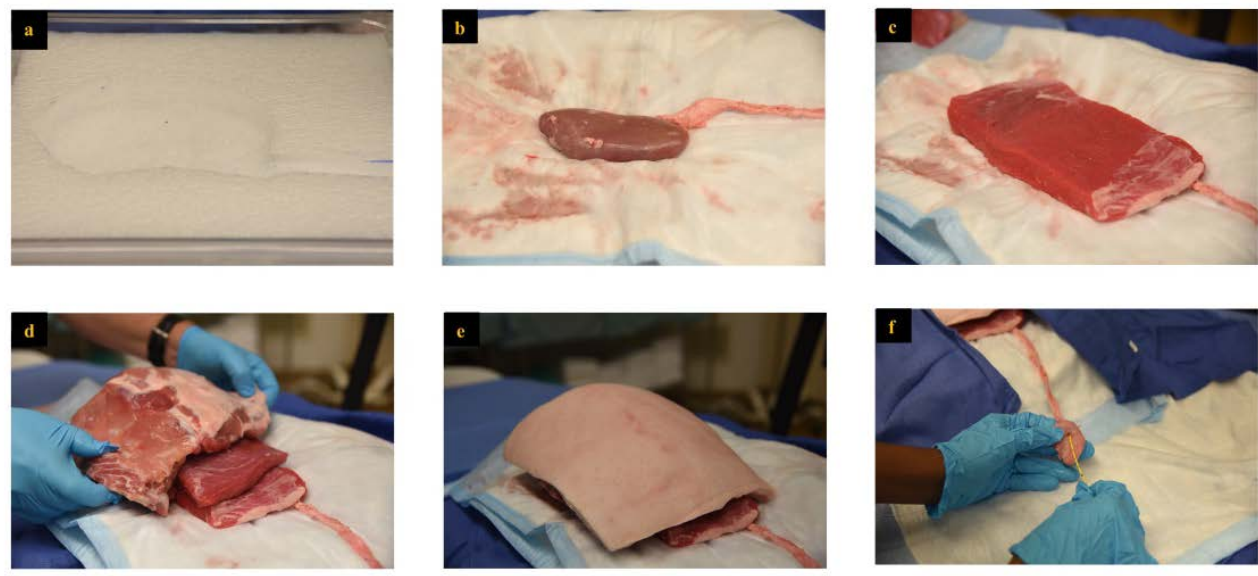

Fig. 2. Ex-vivo PCNL access using partial pig model. (a) Complete setup of partial pig model: kidney and ureter are covered with additional layers of tissue. (b) Trocar needle is introduced into the partial pig model. (c) Insertion of guide wire into posterior calyx through the trocar needle under fluoroscopic guidance. (d) A high pressure nephrostomy balloon catheter is used to dilate the nephrostomy tract. (e) Access sheath is guided over the nephrostomy balloon. (f) Porcine kidney with ureter and percutaneous sheath in the collecting system.
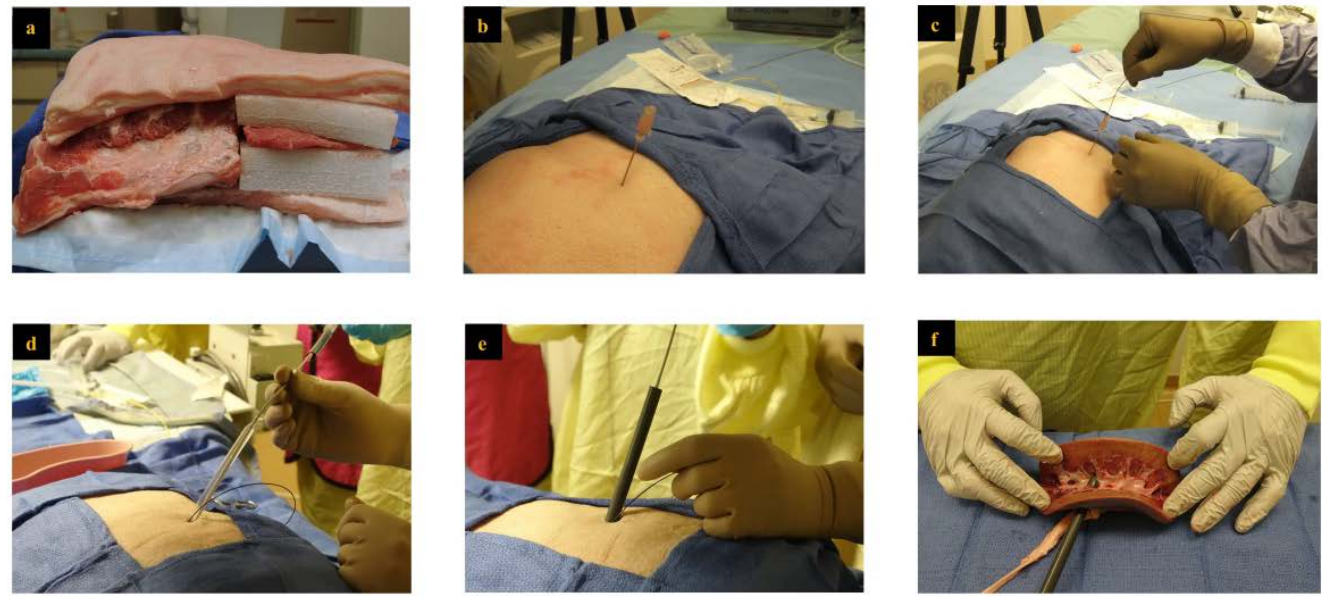
Fig. 3. Fluoroscopic images of percutaneous nephrolithotomy access using partial pig model. (a) Retrograde pyelogram is used to identify calyces. (b) A trocar needle is inserted and advanced to a posterior renal calyx. (c) Through the needle, a guidewire is introduced into the calyx and subsequently, (d) into the ureter. (e) A high pressure nephrostomy balloon catheter was inserted and used to dilate the nephrostomy tract. (f) Following dilation, renal sheath was inserted to allow access for percutaneous nephrolithotomy. Picture demonstrates ability to obtain multiple accesses with this model.
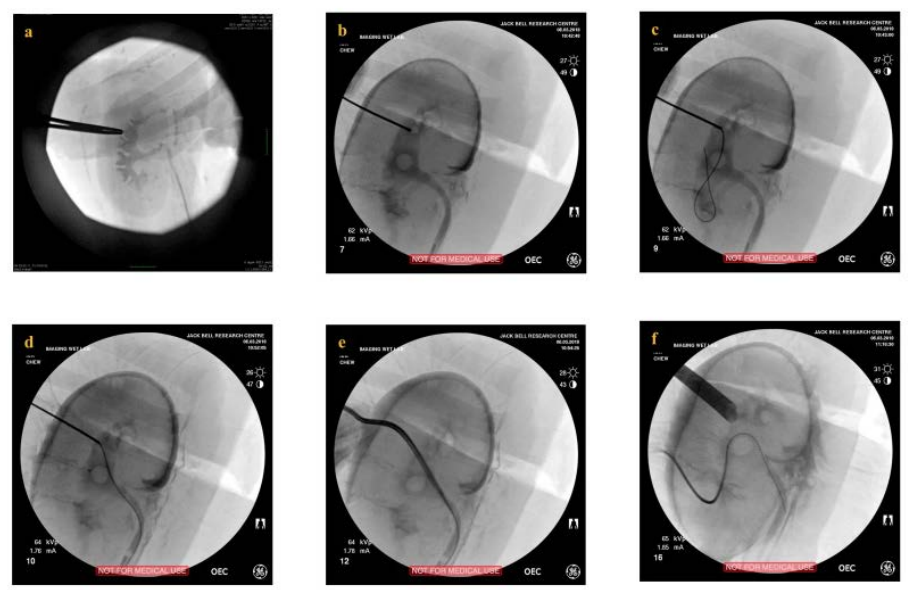

Fig. 4. Setup of silicone model for teaching of percutaneous access. Backlit silicone model containing simulation collecting system is used for practicing targeting, puncture, and dilation. This figure was provided courtesy of Cook Medical.

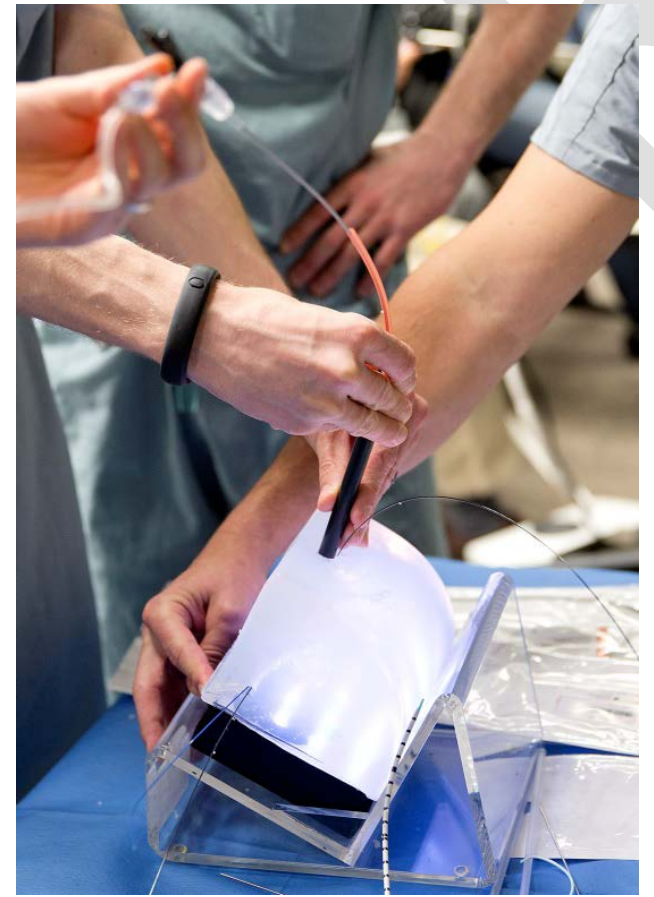




\begin{tabular}{|c|c|c|c|c|c|}
\hline \multicolumn{6}{|l|}{ Silicone model group $(n=14)$} \\
\hline Question & $\begin{array}{l}\text { Strongly } \\
\text { agree }\end{array}$ & Agree & Neutral & Disagree & $\begin{array}{l}\text { Strongly } \\
\text { disagree }\end{array}$ \\
\hline $\begin{array}{l}\text { Have you obtained your own PCNL access } \\
\text { prior to this course?* }\end{array}$ & $7 \%$ & - & - & - & $93 \%$ \\
\hline This model was easy to use. & $50 \%$ & $29 \%$ & $7 \%$ & $7 \%$ & $7 \%$ \\
\hline $\begin{array}{l}\text { The model increased my understanding of } \\
\text { the anatomy. }\end{array}$ & $7 \%$ & $50 \%$ & $29 \%$ & $0 \%$ & $14 \%$ \\
\hline $\begin{array}{l}\text { The model was similar to what I would } \\
\text { expect in a human patient. }\end{array}$ & $15 \%$ & $8 \%$ & $23 \%$ & $54 \%$ & $0 \%$ \\
\hline The puncture needle was easy to use. & $64 \%$ & $29 \%$ & $7 \%$ & $0 \%$ & $0 \%$ \\
\hline $\begin{array}{l}\text { I have a better knowledge of the steps to } \\
\text { obtaining PCNL access. }\end{array}$ & $36 \%$ & $29 \%$ & $21 \%$ & $7 \%$ & $7 \%$ \\
\hline $\begin{array}{l}\text { I feel more confident in my ability to gain } \\
\text { PCNL. }\end{array}$ & $21 \%$ & $29 \%$ & $29 \%$ & $14 \%$ & $7 \%$ \\
\hline This was a worthwhile experience. & $57 \%$ & $14 \%$ & $14 \%$ & $7 \%$ & $7 \%$ \\
\hline
\end{tabular}

${ }^{*}$ For question answered as YES or NO: YES recorded as Strongly agree, and NO recorded as Strongly disagree. PCNL: percutaneous nephrolithotomy. 


\section{Table 2. Summary of partial pig model questionnaire results}

Partial pig model group $(n=14)$

\begin{tabular}{|l|c|c|c|c|c|c|}
\hline Question & $\begin{array}{c}\text { Strongly } \\
\text { agree }\end{array}$ & Agree & Neutral & Disagree & $\begin{array}{c}\text { Strongly } \\
\text { disagree }\end{array}$ & $\begin{array}{c}\text { p (vs } \\
\text { silicone) }\end{array}$ \\
\hline $\begin{array}{l}\text { Have you obtained your own PCNL access } \\
\text { prior to this course? }\end{array}$ & $15 \%$ & - & - & - & $85 \%$ & 0.53 \\
\hline This model was easy to use. & $43 \%$ & $50 \%$ & $7 \%$ & $0 \%$ & $0 \%$ & 0.92 \\
\hline $\begin{array}{l}\text { The model increased my understanding of } \\
\text { the anatomy. }\end{array}$ & $50 \%$ & $50 \%$ & $0 \%$ & $0 \%$ & $0 \%$ & $<0.01^{*}$ \\
\hline $\begin{array}{l}\text { The model was similar to what I would } \\
\text { expect in a human patient. }\end{array}$ & $21 \%$ & $79 \%$ & $0 \%$ & $0 \%$ & $0 \%$ & $<0.01^{*}$ \\
\hline The puncture needle was easy to use. & $36 \%$ & $36 \%$ & $28 \%$ & $0 \%$ & $0 \%$ & 0.1 \\
\hline $\begin{array}{l}\text { The guide wire was easy to use. (only asked } \\
\text { to partial pig model group) }\end{array}$ & $64 \%$ & $29 \%$ & $7 \%$ & $0 \%$ & $0 \%$ & N/A $^{*}$ \\
\hline $\begin{array}{l}\text { I have a better knowledge of the steps to } \\
\text { obtaining PCNL access. }\end{array}$ & $93 \%$ & $7 \%$ & $0 \%$ & $0 \%$ & $0 \%$ & $<0.01^{*}$ \\
\hline $\begin{array}{l}\text { I feel more confident in my ability to gain } \\
\text { PCNL. }\end{array}$ & $57 \%$ & $29 \%$ & $14 \%$ & $0 \%$ & $0 \%$ & $0.02^{*}$ \\
\hline This was a worthwhile experience. & $86 \%$ & $14 \%$ & $0 \%$ & $0 \%$ & $0 \%$ & $<0.01^{*}$ \\
\hline
\end{tabular}

${ }^{\dagger}$ For questions answered as YES or NO: YES recorded as Strongly agree, and NO recorded as Strongly disagree. ${ }^{*}$ Statistically significant. PCNL: percutaneous nephrolithotomy.

\begin{tabular}{|l|c|}
\hline Table 3. Estimated cost of ex-vivo pig model \\
\hline Component & Cost (\$CDN) \\
\hline Porcine rib block & 113 \\
\hline Porcine skin & 29 \\
\hline Porcine kidney & 20 \\
\hline Flank steaks & 18 \\
\hline Packing charge & 36 \\
\hline Total costs & $\mathbf{2 1 6}$ \\
\hline
\end{tabular}

